\title{
Tendencies and Preferences of Choosing Information Sources in Academic Learning: A Case of Native Hebrew and Native Arabic Speakers in Israel
}

\author{
PhD. Moti Merdler* \\ Western Galilee College, Department of education \& H.R. management, Akko, Israel \\ Email: motim@wgalil.ac.il ,POB 2125, Akko 24121 \\ PhD. Yehuda Peled \\ Western Galilee College, Information Studies Department, Akko, Israel \\ Email: yehudap@wgalil.ac.il, POB 2125, Akko 24121; *Corresponding author
}

\section{Doi:10.5901/jesr.2016.v6n1p39}

\section{Abstract}

In this article, we describe a study which examined factors that dominate the preferences of Israeli native Arabic speaking (NAS) students and those of Israeli native Hebrew speaking (NHS) students for choosing digital, printed, written, or orally conveyed information sources for academic learning. The study included 173 students (109 NHS; 64 NAS) from two academic institutions located in northern Israel who participated in the same two annual academic courses. We examined the perceived attribution of eight different information sources common in academic learning and 15 information source criteria that are relevant for the NAS and NHS students' preferences. We conclude that differences in perception of scope, depth, accessibility, trustworthiness, clarity, and especially the perceived ability of cognitive processing from various information sources can explain the "Digital Divide" between NAS and NHS Israeli students in the context of evaluating the contribution of different information sources for their learning. Thus, a techno-socio-cultural Second Order Digital Divide emerges.

Keywords: Information characteristics, Digital divide, Decision making, Native language cultural learning effect.

\section{Introduction}

As adults, we perceive the world, and we conceive and learn about its nature by using the information to which we have been exposed and by the manner in which we process it. Ostensibly, techno-economic globalization, by creating an abundance of highly accessible information sources to diverse populations, was expected to narrow the gap in knowledge-based information processing between minorities and socio-economic dominant populations. However, in the last decade or so, in many minority socio-environments, this narrowing has not been achieved (Ganayem, Rafaeli, \& Azaiza, 2009). Although "digital readiness" is widespread, socio-cultural and other factors seem still not to support such technological readiness among minorities (Habib, Johannesen, \& Øgrim, 2014). Digital readiness is the extent to which a population, a group, or a person has the potential to acquire and integrate cognitive skills in an instrumental digital environment (Horrigan, 2014). Lack of such readiness creates a "digital divide" that is commonly susceptible to differences of gender, education, age, language skills and socio-economic status. One of the important aspects of the study about the impact of this digital divide on integrating minorities, and one that has hardly been studied, is how minorities perceive, evaluate, and implement information sources and information in general, particularly in comparison to well-based dominant socio-economic populations in a society.

Therefore, the purpose of this study was to return to the fundamental issues and raise some rather basic questions concerning the distinctive characteristics of a specific minority regarding their information perception. We assumed that answers to such questions would shed light on the difficulty of narrowing the digital divide between minorities and majorities, inasmuch as the latter are the more technologically advanced population.

The primary and most common way in which most of the public engage with information in a scholarly mode is through learning in order to acquire education. Meaningful learning is a process that has critical implications that extend beyond merely acquiring knowledge. It is the major factor in creating differences in income and standards of living among various sectors of a population. In Israel, this factor is dominant among women, both ultra-orthodox NHS and NAS (Yashiv, 2011), hence the importance of the issue and the contribution to the knowledge on the subject. 


\section{Theoretical Framework and Literature Review}

Previous studies have shown some of the factors that directly or indirectly affect difficulties in using digital information sources as leverage for academic orientation, and enhance the preference for the use of certain information sources, especially by the NAS public. These factors can be divided into two main groups. First, there are factors based on the degree of implementation and assimilation of technology (computer ownership, browsing speed, etc.). These are monotype factors, i.e. indexes that focus on one issue that we wish to explain (Barzilai-Nahon, 2006). Second, there are contextual factors, which are comprehensive and based on the satisfaction of users' individual and multiple needs in their operative environments (communication, social networks, cultural interests, etc.).

The main factors associated with assimilation and implementation are related to accessibility and availability and are usually referred to as factors causing a "first-order digital divide" ("accessing divide") (Zeng, 2011). These factors include the following: (1) owning a computer; (2) having an Internet connection (Mesch \& Talmud, 2011); (3) the specific position on Roger's innovation distribution curve; (4) socio- economic status; (5) gender; (6) geographic location; (7) religion; (8) age; (9) education (Ganayem, Rafaeli, \& Azaiza, 2009); (10) the extent of usage and its characteristics (Boniel-Nissim \& Daw, 2009), and other similar factors (Barzilai-Nahon, 2006). Although these factors might explain the digital divide in terms of accessibility and availability of interactive-informative digital media, they still cannot explain the differences in dispositions and tendencies of using these digital media among NAS and NHS students, when their access to digital information sources is fairly equal. In other words, when conditions for technological assimilation are equal, what can explain the differences in assessment, preference, and use of information sources between NHS and NAS students?

Among the contextual factors causing a second order digital gap ("usage divide") (Zeng, 2011) that may explain a preference for the use of digital information (or other information sources), we can find the following:

- differences in exposure to the Internet and digital media in the workplace that create negative attitudes towards information technology and mastery level English (Mesch \& Talmud, 2011),

- the possibility of circumventing cultural prohibitions of interpersonal relationships,

- the tendency to adopt western cultural values (Boniel-Nissim \& Daw, 2009),

- the need for accessing the latest information not only through the Israeli media (Kabha, 2007), and

- satisfying intellectual needs such as developing critical thinking and professional development.

Also we can find factors concerning the use of language (Hebrew) as a tool for instrumental integration, sociolinguistic gender tendencies in use of communication technology (Gefen, Geri, \& Paravastu, 2007), and the socialcultural meaning of religious affiliation and its power in the sense of hierarchy, patriarchy, discipline, and seclusion (Barzilai-Nahon \& Barzilai, 2005).

Both in theory and in practice, mastery of the language of instruction, especially when it is a second language, seems to be the dominant contextual factor. The level of such mastery should affect the preference for digital, written, printed, or oral information sources equally among speakers of Hebrew as a second language who have English as a third language. Although a certain gap in learning skills seems likely to cause difficulties for acquiring new information between NHS and NAS students when the information and its source is in Hebrew, this gap is actually not explained predominantly by the level of Hebrew mastery (Miller \& Peleg, 2010). Even after gaining sufficient mastery to enable learning, the second language speakers still show no significant change in the level of textual understanding.

In a comprehensive experiment, Miller and Peleg (2010) examined students' ability to learn Hebrew words, both those that have meaning and nonsense words (meaningless strings of letters). They found that native Hebrew speakers' ability to learn the words were higher than the ability of native Arabic speakers. Miller and Peleg concluded that language mastery do not solely explain learning ability when learning is not in the native language. Miller and Peleg offered two explanations for this finding, one cognitive and one emotional. The first explanation suggests a difference in working memory between NHS and NAS, as a minority population. They relied on similar findings among populations who speak proximate languages (the researchers have found the proximity of Hebrew and Arabic as Semitic languages to be similar to the proximity of German, Dutch, and Norwegian as Germanic languages) (Van den Noort, Bosch, \& Hugdahl, 2006). The second explanation they offered is anxiety due to using a second language in studying (Abu Rabia, 2004).

The reasons for the preference of information sources seem then to be based on a variety of factors not necessarily related to distribution, accessibility, or availability of the medium. The question, therefore, is which characteristics of the information perception are the factors that influence decision-making about the information source selection and its use?

In recent years, the perception of information has changed paradigmatically. Changes in availability, accessibility, 
updateability, and contemporariness of harmonic digital information sources may cause the printed and disharmonic information sources to be obsolete. The argument that information perception is changing paradigmatically is also based on the observation that the cognitive abilities and skills needed to process information from digital vs. from printed sources have major differences (Eshet \& Amichai, 2004), especially in terms of the complex cognitive skills necessary for digital information processing (for example: hyper-texts). On the other hand, the common tendency is to attribute higher reliability to printed information because it is formal and (usually) distributed from an expert source. This is especially true in an environment of uncertainty about the subject at hand (Tiedens \& Linton, 2001).

It is fair to assume that in cases where learning topics do not raise the individual's emotions, cognitive aspects will be the main factors in determining the attitude toward a source of information and how one perceives it. Thus, by aiming to maximize the expected utility from using the information source, the user will consider the value of the expected information relative to the resources needed for extracting it from the source (Venkatesh \& Morris, 2000). Although this perception of benefit varies with experience, one can still reasonably estimate the chances of success in using the specific source by referring to experts' and experienced users' evaluations (Gershoff, Broniarczyk, \& West, 2001).

Although people tend to select an information source by the possibility of maximizing its utility in learning (as perhaps in general usage), the abundance and availability of digital information creates difficulties for such maximization. This phenomenon is well known from the Minnesota experiments conducted in the early 1970s, and its essence is that information richness allows the maximizing of information utility, but it also induces an association of uncertainty, thus resulting in a lack of confidence in the selection of information sources (Banker \& Kauffman, 2004). Moreover, this conflict causes a heuristic bias in the selection of sources because the abundance of choices generates the thought of a potentially better choice than the one chosen, imposing more responsibility on the student's source selection. Therefore, students may often regret their choices; no matter how excellent the choices were, because the profusion of sources creates the appearance of other options that might have been better (Schwartz, 2005). For a better understanding of information source selection and the decision-making process involved, we need to examine the dominant criteria by which information sources are evaluated in academic learning.

For this study of evaluating information sources in the academic context at the beginning of the digital age, we have adapted printed information criteria for evaluation to apply to digital information. Early in digital age, Alexander and Tate (1996) divided the criteria for evaluating information into five groups: authority, objectivity, scope, contemporariness, and accuracy. A decade later, the perception of assessing digital information source had somewhat changed. The indicators of scope and accuracy (content criteria) became less important, while information source criteria (authority, objectivity, and, to a certain degree, contemporariness) became the main indicators while distinguishing between the publisher's and the authors' authority (Knight\& Burn, 2005). This change may be an attempt to deal with multiple sources of digital information (academic and other). The assessment of information quality is mainly an evaluation of the source's "status" (the authors' and the publisher's) and a relatively superficial evaluation of the information itself (Towlson, Leigh, \& Mathers, 2009).

Therefore, the questions to which we aimed this study are as follows:

- What are the differences among NHS and NAS students regarding their preferences for educational information sources? and

- What are the differences in the criteria for preferred educational information sources between NHS and NAS students?

\section{Research Methodology}

In order to answer these questions, we constructed an attitude and perception questionnaire that relates information source characteristics to learning. Data collected from a student focus group from the Department of Information Science indicate a reasonable reliability of the questionnaire (Cronbach's alpha was between 0.80 to 0.87 among NAS and NHS). The age range of the participants was 18 to 30 (no significant age-related differences were found in positions, perceptions, or preferences for information sources). After analyzing the pilot questionnaire items for reliability and validation, we removed items that turned out to have low reliability. After this step, the questionnaire given to the whole population contained the following criteria and characteristics (independent factors):

- Availability of information source: the ease of obtaining the source of information (5=very easy, $1=$ =very difficult).

- Accessibility of information from the source: the ease of obtaining information from the source, once the source is available (5=very easy, $1=$ =very difficult). 
- Substantiation of information about the topic: the ease of understanding the bases of information and knowledge in this source.

- Relevance of the information: the extent to which the information presented is relevant and related to the topic.

- Interest and curiosity that information raises: information and knowledge from this source is unique and stimulates a desire to know more.

- Information trustworthiness: the extent to which information is accurate and based on well-defined terms (5=very well based and accurate, 1=doubtful establishment).

- Contemporariness: the extent to which information is contemporary or old (5=very contemporary, $1=0$ obsolete).

- Scope of information: the physical size of the information, e.g. number of words, paragraphs (5=very large, $1=$ very small).

- Comfort level of information processing: how convenient it is to work with the information while given a cognitive task, such as searching, sorting, comparing, or solving problems (5=very easy, 1=very difficult).

- Comfort level of additional information mining: the convenience of searching more information in this medium (5=very convenient, $1=$ =very difficult).

- The depth and detail of information: the extent to which information contains extensive details and meanings ( $5=$ very detailed, $1=$ very superficial).

- Comfort of reading the information: how comfortable it is to read the text, charts, etc. (5=very easy, $1=v e r y$ difficult).

- Comfort of using the information for writing: how convenient it is to write on the basis of the given information (5=very easy, $1=$ =very difficult).

- Convenient storage of information: how convenient it is to keep the information for further use (5=very easy, 1=very difficult).

- Clarity of information: the extent to which the presented information is clear and obvious ( $5=$ =very easy, $1=v e r y$ difficult).

In addition, some other general independent factors were measured: age, gender, residency (rural, urban - small, large) and mastery of English (1-5).

The dependent factor that was examined:

- Learning consolidation priority: to what extent would you prefer to use this information source in learning? (5=highly preferred, $1=$ =mostly avoided).

The students' evaluations of the information source criteria were examined for eight information source domains: (1) online scientific journal articles; (2) online newspaper articles; (3) scientific videotapes/DVDs or YouTube-type videos of hands-on experiments; (4) blog posts, wall posts, or other social networks postings (Facebook, Twitter, etc.); (5) digital encyclopedias or dictionaries; (6) scientific-based printed articles or books; (7) summaries and notes of course lectures; and (8) trustworthy individuals who I believe understand the issue.

In order to answer the research questions, we applied two data analyses. First, we implemented independent Ttests for comparing NAS and NHS students' perceived value of various domains of information sources. The second analysis was a stepwise multi-variance regression to establish the main factors influencing the preferred information sources chosen for learning, among NAS and NHS students.

\section{Findings}

The most conspicuous finding was that NAS students did not appreciate online scientific journal articles as one of the main information sources needed for academic learning, whereas NHS students regarded this information source domain as one of the two most appreciated (NAS average 3.33, NHS average 4.12). This finding is intriguing because English language mastery was not found as an influencing factor, although English is not a native language for either population. We made a similar observation regarding digital encyclopedias or dictionaries as a source for learning (NAS average. 3.11, NHS average. 3.66, sig. <0.001). A second, but rather expected and less salient finding than the first, is that NHS students relied on printed articles or books from scientific origins as a dominant source more than did the NAS students (NAS average. 3.89, NHS average 4.19, P-value $=0.067$ ).

The NAS students seemed to rely on trustworthy individuals who they believed understand the issue as a compensating factor for presumed difficulties in relying on scientific origins, digital or otherwise, whereas NHS students 
did not regard such a source as a profound or available source for learning (NAS average. 3.81, NHS average 3.40, seg.>0.05).

Table 1: NAS and NHS students' preferences for using different information sources.

\begin{tabular}{|c|c|c|c|c|c|c|c|c|}
\hline & 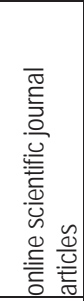 & 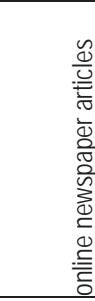 & 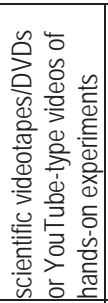 & 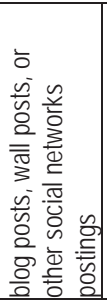 & 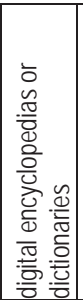 & 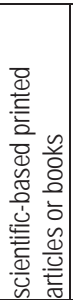 & 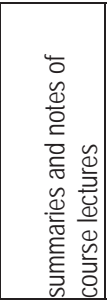 & 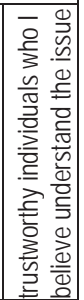 \\
\hline $\begin{array}{l}\text { NAS (N=64) } \\
\text { Mean (SD.) }\end{array}$ & $\begin{array}{c}3.33 \\
(1.13) \\
\end{array}$ & $\begin{array}{c}3.08 \\
(1.09)\end{array}$ & $\begin{array}{c}3.35 \\
(1.11) \\
\end{array}$ & $\begin{array}{c}2.52 \\
(1.09) \\
\end{array}$ & \begin{tabular}{|c|}
3.11 \\
$(1.17)$ \\
\end{tabular} & $\begin{array}{c}3.89 \\
(0.96) \\
\end{array}$ & $\begin{array}{c}3.98 \\
(1.14) \\
\end{array}$ & $\begin{array}{c}3.81 \\
(1.12) \\
\end{array}$ \\
\hline NHS (N=109) Mean (SD.) & $\begin{array}{c}4.12 \\
(0.83)\end{array}$ & $\begin{array}{c}3.32 \\
(0.95)\end{array}$ & $\begin{array}{c}3.56 \\
(1.04)\end{array}$ & $\begin{array}{c}2.45 \\
(1.12)\end{array}$ & $\begin{array}{c}3.66 \\
(1.15) \\
\end{array}$ & $\begin{array}{c}4.19 \\
(0.79) \\
\end{array}$ & $\begin{array}{c}3.97 \\
(0.95)\end{array}$ & \\
\hline 保 & $<0.001$ & Non sig. & Non sig. & Non sig. & $<0.01$ & $<0.1$ & Non sig. & $<0.0$ \\
\hline
\end{tabular}

For a better understanding of the causes and factors upon which NAS and NHS students prefer specific source domains of information for learning, we analyzed two salient types of information sources for the independent factors that dominated the selections among our participants. We examined the selections of online scientific journal articles [Table 2] and trustworthy individuals [Table 3] because they were the most influential information criteria.

Table-2 (a): Factors effecting online scientific journal articles as a source preference among NAS students.

\begin{tabular}{|c|c|c|c|c|c|c|c|c|}
\hline & 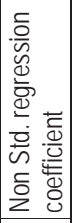 & & 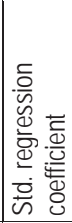 & 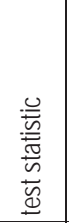 & & \multicolumn{3}{|c|}{ 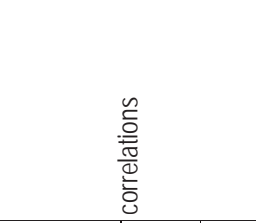 } \\
\hline independent variables & B & Std. Error & Beta & $\mathrm{t}$ & Sig. & Zero-order & Partial & Part \\
\hline Substantiation of information about the topi & 0.375 & .120 & 0.449 & 3.138 & $<.001$ & 4.449 & .449 & .449 \\
\hline
\end{tabular}

Note: Multiple-regression (stepwise method) for factors effecting online scientific journal articles source preference for learning among NAS students ( $N=64)$. Dependent variable: online scientific journal articles as a source for learning.

In light of the findings presented in Tables 1 and 2(a), NAS students seem likely to avoid using online scientific journal articles unless the information from this source were well substantiated for the topic at hand $(r=.449)$.

Table-2 (b): Factors affecting online scientific journal articles source preference for learning among NHS students.

\begin{tabular}{|c|c|c|c|c|c|c|c|c|}
\hline & 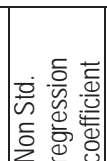 & & 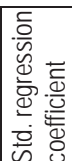 & 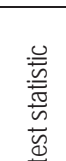 & & \multicolumn{3}{|c|}{ 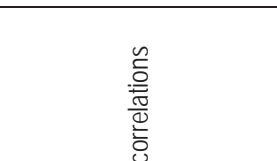 } \\
\hline independent variables & $\mathrm{B}$ & Std. Error & Beta & $\mathrm{t}$ & Sig. & Zero-order & Partial & Part \\
\hline Comfort level of information processing & 270 & 0.100 & 0.298 & 2.698 & 0.009 & 0.526 & 0.297 & 0.242 \\
\hline Substantiation of information about the topic & 205 & 0.082 & 0.255 & 2.512 & 0.014 & 0.471 & 0.279 & 0.225 \\
\hline Comfort level of additional information mining & 201 & 0.098 & 0.233 & 2.059 & 0.043 & 0.512 & 0.231 & 0.185 \\
\hline
\end{tabular}

Note: Multiple-regression (stepwise method) for factors effecting online scientific journal articles source preference for learning among NHS students ( $N=109)$. Dependent variable: online scientific journal articles as a source for learning. 
NHS students' reasons for choosing online scientific journals as information sources are complex. They mostly valued the characteristics of the comfort level of information processing ( $r=.242)$, the substantiation of information about the topic (.225), and the comfort level of additional information mining (0.185) [Table 2] imbedded in online scientific journal articles.

Table-3 (a): factors effecting the reliance on trustworthy individuals who understand the issue as a source preferred for learning among NAS students

\begin{tabular}{|c|c|c|c|c|c|c|c|}
\hline & 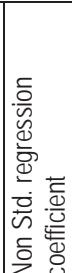 & & 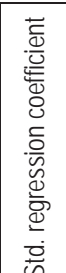 & 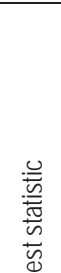 & & & $\frac{\mathscr{0}}{\frac{\mathscr{0}}{ \pm}}$ \\
\hline independent variables & $B$ & Std. Error & Beta & t & Sig. & Zero-order & Partial Part \\
\hline Availability of the information source & 0.720 & 0.109 & 0.686 & 6.599 & $<<0.001$ & 0.739 & \begin{tabular}{|l|l|l|}
0.722 & 0.665 \\
\end{tabular} \\
\hline Interest and curiosity that the information raises & 0.244 & 0.114 & 0.223 & 2.144 & 0.038 & 0.388 & 0.3210 .216 \\
\hline
\end{tabular}

Note: Multiple-regression (stepwise method) for factors affecting the reliance on trustworthy individuals who understand the issue as a source preferred for learning among NAS students. ( $\mathrm{N}=64)$. Dependent variable: reliance on trustworthy individuals who understand the issue as an information source.

NAS students seemed likely to approach a scholar for obtaining information due to two main factors: availability of the scholar ( $r=.665)$ and intrigue level of the topic - to some extent, the students' interest and curiosity (.216) [Table 3(a)]. Among NHS students, specific attention to an individual scholar's knowledge and information depends mainly on the direct relevance to the topic or issue at hand $(r=.431)$ [Table 3(b)]. While NAS students sought for available individuals who possess scholarly or expert knowledge, and they also seemed to look for the scholars' opinion and guidance on the topic the students' were learning about, the NHS students tended to refer to those presumed scholars or experts according to relevance.

Table-3 (b): factors effecting the reliance on a trustworthy individual who understands the issue as a source preferred for learning among NHS students.

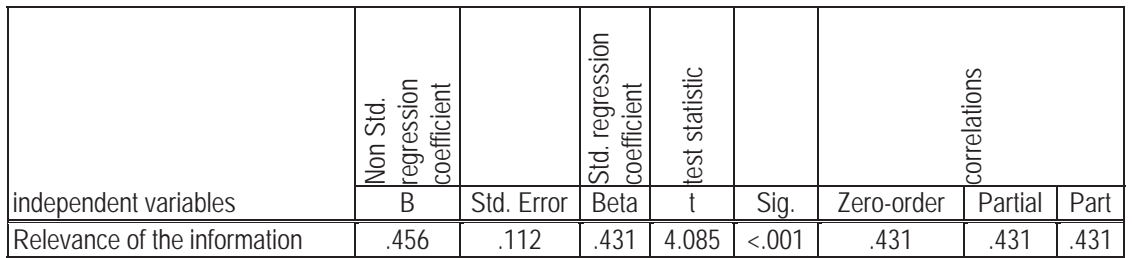

Note: Multiple-regression (stepwise method) for factors effecting the reliance on a trustworthy individual who understands the issue as a source preferred for learning among NHS students. $(\mathrm{N}=109)$. Dependent variable: reliance on a trustworthy individual who understands the issue as an information source.

\section{Discussion}

In light of the findings presented in Tables 1 and 2(a), the NAS students seemed likely to avoid using online scientific journal articles for learning, unless the information from the source were well substantiated for the topic at hand. As this domain of information becomes more common for academic learning, educators should concern themselves with the issue of substantiating online information sources. The issue of substantiation, or rather students' perception of substantiation, should be explored further for what sort of substantiation is perceived as convincing enough, beyond rigorous study methodologies. Among the NHS students, this factor also affected using online articles, but it was combined with two other factors: their comfort level with information processing and their comfort level with additional 
information mining. Although for both student populations the correlations were medium-low and thus have a limited explanatory value $\left(20 \%<\mathrm{R}^{2}<26 \%\right)$, still the correlations reveal a critical perception about students' attitude towards online scientific articles.

The NAS students tended to seek information that was processed and constructed into a body of knowledge and that was orally conveyed by a scholar, whenever he or she was available and raised interest and curiosity $\left(40 \%<R^{2}<48 \%\right.$, Table $\left.3(a)\right)$. This reliance on a scholar as a mediator of information was more prominent among NAS than NHS students (sig. $<0.05$, Table 1). However, NHS students will consider using an orally-presenting scholar as a source if the information is relevant to learning $\left(R^{2}=19 \%\right.$, Table $\left.3(b)\right)$. Those findings, among others, led us to construct two sets of explanatory perspectives for the "second-level digital divide' (Zeng, 2011) among minorities, both global and local.

From the global perspective of socio-cultural "digitally divided" minorities, the NAS students' reliance on an information and knowledge mediator is not merely a technological one. Minorities, native or immigrant, rely on trustworthy mediators and facilitators whom the minorities consider to be part of the well-established techno-social population. This observation seems to be common among many multicultural societies in the USA with differences between particular minorities (Kress, 2009), in India concerning the Muslim minorities (The Report on the National Consultative Summit, 2011), and in Finland concerning international students (Habib, Johannesen, \& Øgrim, 2014). Habib, Johannesen, and Øgrim (2014) captured the essence of this goal of encountering the problem thus:

"Technology as a socio-material assemblage may encapsulate cultural codes that can be alienating for international students and that there is a need to 'open the black boxes' of technology to cater for the needs of international students" (p. 196).

From the local Israeli perspective, the differences between NHS and NAS students can be explained by two sets of reasons: socio-educative and cognitive-linguistic norms. The socio-educative reasons could include previous findings that NAS K-12 students study in an authoritative and traditional environment that emphasizes the need to respect the wisdom of the elders. Thus, the learning environment does not prompt curiosity and does not encourage the students to ask questions, to research, or to be creative (Abed, \& Dori, 2013). Furthermore, the NAS undergraduates seem to learn patterns in a similar way to their learning patterns in the K-12 system (Peled \& Khaldi, 2013), which Abed \& Dori (2013) described as a simple and linear learning pattern. The cognitive-linguistic set of reasons can suggest differences in working memory (Miller \& Peleg, 2010), deficiency in using the right hemisphere while reading in one's native language and especially when evoked bilaterally (Ibrahim \& Eviatar, 2012) or decision making preferences that are subjected to "avoiding the worst" while managing a tangling abundance of information (Merdler, 2012).

\section{Conclusion}

The practical implication of these findings may be a contribution to maximizing minorities' learning potential. We suggest that in order to utilize the minorities' fullest learning potential, socio-technological-cultural mediation is needed to bridge the digital divide.

It is commonly known that minorities, may they be native or immigrant by their nature, tend to preserve their own cultural identity. According to the reviewed literature on the issue and our research findings, some changes in the cultural way by which information technology is used by minorities should be made to maximize their assimilation in the learning and workplace environments. Encountering such goal should not be the sole concern of those minorities but rather the concern of the well-established local majority. Therefore, well prepared and motivated individual, within those majorities should be their mediators, educators and assimilators in the field of information technology usage, at the workplace as well as at educational institutes.

The study uses a multi-level-domain questionnaire which was constructed according to the known and reasonable issues and characteristics appearing in the literature, but by all means it may not be the only ones effecting information source preference. Therefore, we suggest that further research should illustrate the actual information preferences when students value and choose information sources while learning, presumably by an experiment and/or qualitative research methodologies. While the study external validity is limited for its small and rather homogenous sample, still the internal validity is more than adequate since it reflects the local (galilee-northern Israel) digital divide student's entanglement. Therefore, the socio-cultural complexities of effective information technology assimilation should reflect both local uniqueness as well as global tendencies. 


\section{References}

Abed, A. \& Dori, Y. (2013). Inquiry, chemistry understanding levels, and bilingual learning. educación química, 24(1), 37-43.

Abu Rabia, S. (2004). Teachers' role, learners' gender differences, and FL anxiety among seventh-grade students studying English as an FL. Educational Psychology, 24, 712-724.

Alexander, J. \& Tate, M. (1996). Teaching critical evaluation skills for World Wide Web resources. Computers in Libraries, 16(10), 49-55.

Banker, R.D. \& Kauffman, R. J. (2004). The Evolution of Research on Information Systems: A Fiftieth-Year Survey of the Literature in Management Science. Management Science, 50(3), 281-297.

Barzilai-Nahon, K. \& Barzilai, G. (2005). Cultured technology: Internet and religious fundamentalism. The Information Society, 21, 2540.

Barzilai-Nahon, K. (2006). Gaps and bits: Conceptualizing measurements for digital divide/s. The Information Society, 22(5), $269-278$.

Boniel-Nissim, M. \& Daw, S. (2009). Virtual culture in a multicultural society. Et Asadde, 3, 17-23. (Hebrew).

Eshet, Y.A. \& Amichai, Y. (2004). Experiments in digital literacy. Cyberpsychology \& Behavior, 7(4), 421-428.

Ganayem, A., S. Rafaeli, \& F. Azaiza. 2009. Digital divide: Internet usage in the NAS sector in Israel. Megamot, 36, 164-196. (Hebrew).

Gefen, D., Geri, N., \& Paravastu, N. (2007). Vive la Différence: The cross-culture differences within us. International Journal of eCollaboration, 3(3), 1-14.

Gershoff, A.D., Broniarczyk, S.M., \& West, P.M. (2001). Recommendation or evaluation? Task sensitivity in information source selection. Journal of Consumer Research, 28, 418-437.

Habib, L., Johannesen, M., and Øgrim, L. (2014). Experiences and challenges of international students in technology-rich learning environments. Educational Technology \& Society, 17(2), 196-206.

Horrigan, J. B. (2014). Nearly One-Third of American Adults Lack Digital Readiness. Targeted News Service. http://www.siliconflatirons.org/documents/conferences/2014-04-29.

Ibrahim, R. \& Eviatar, Z. (2012). The contribution of the two hemispheres to lexical decision in different languages. Behavioral and Brain Functions 2012, 8(3), 1-7.

Kabha, M. (2007). Network without Borders: Using the Internet and online journalism in the NAS population in Israel, in: T. Schwartz Altshuler (Ed.), Press Dot.com: online journalism in Israel (pp. 177-196). Jerusalem: The Israel Democracy Institute and Burda Center for Innovative Communications at Ben - Gurion University. [Hebrew]

Knight, S. \& Burn, J. (2005). Developing a framework for assessing information quality on the World Wide Web. Informing Science Journal, 8, 159-172

Kress, T.M. (2009). In the shadow of whiteness: (Re)exploring connections between history, enacted culture, and identity in a digital divide initiative. Cultural Studies of Science Education, 4, 41-49.

Merdler, M. (2012). Information Characteristics Perceptions and Information Source Preferences in Learning among Jewish and Arab Students in Israel. Proceedings from ICERI2012: 5th International Conference of Education, Research and Innovation, Madrid, Spain: IATED.

Mesch, G.S. \& Talmud, I. (2011). Ethnic differences in Internet access: The role of occupation and exposure. Information, Communication \& Society, 14(4), 445-471.

Miller, P. \& Peleg, O. (2010). Doomed to read in a second language: Implications for learning. Journal of Psycholinguist Research, 39 , $51-65$.

Peled, Y. \& Khaldi, S. (2013). Are discrimination, survival and tradition sufficient argument for academic dishonesty? Discrimination, survival and tradition as argumentation for academic dishonesty. Educational Practice and Theory, 35(1), 41-61

Schwartz, B. (2005). The paradox of choice: Why more is less. Harper Perennial: New York

The Report on the National Consultative Summit on "Digital Minorities: Leveraging ICT \& Digital Media for Inclusive Development of Minority Communities" at 8th Manthan Award \& Digital Festival for Development on 2nd December, 2011, Digital Empowerment Foundation (DEF) and National Commission for Minorities (NCM). Retrieved from: http://manthanaward.org/IMG/ National\%20 Minority\%20Consulation\%20Report.pdf

Tiedens, L. Z. \& Linton, S. (2001). Judgment under emotional certainty and uncertainty: The effects of specific emotions on information processing. Journal of Personality and Social Psychology, 81(6), 973-988.

Towlson, K., Leigh, M. \& Mathers, L. (2009). The information source evaluation matrix: A quick, easy and transferable content evaluation tool. SCONUL Focus, 15-19. Retrieved from: http://archives.cbc.ca/arts entertainment/media/topics/342-1817/

Van den Noort, M. W., Bosch, P., \& Hugdahl, K. (2006). Foreign language proficiency and working memory capacity. European Psychologist, 11, 289-296.

Venkatesh, V. \& Morris, M.G. (2000). Why don't men ever stop to ask for directions? Gender, social influence, and their role in technology acceptance and usage behavior. Gender in Technology Acceptance and Usage, MIS Quarterly, 24(1), 115-139.

Yashiv, E. (2011). State of the Nation Report - The Macro Picture. In: D. Ben David (Ed.) State of the nation report - society, economy and policy 2010 (pp. 21-48). Jerusalem: Taub Center for Social Policy Studies in Israel.

Zeng, F. (2011). College students' perception of the second-level digital divide: An empirical analysis. Asian Social Science, 7(6), 42-50. 